\title{
Chemical fingerprinting of stellar populations in the Milky Way halo Mei-Yin Chou
}

Institute of Astronomy and Astrophysics, Academia Sinica, Taipei 10617, Taiwan, email: cmy@asiaa.sinica.edu.tw

\begin{abstract}
The idea of "chemically fingerprinting" stars to their birth systems has been discussed over the last decade. Here we present an investigation of the chemical abundance patterns of halo substructures using high-resolution spectra. In particular, we study the abundances of the $\alpha$-like element titanium (Ti) and the s-process elements yttrium (Y) and lanthanum (La) for M giant candidates of the Galactic Anticenter Stellar Structure (GASS, also known as the Monoceros Ring) and the Triangulum-Andromeda (TriAnd) Star Cloud. We apply "chemical fingerprinting" to the GASS/Monoceros Ring and TriAnd Star Cloud, to explore the origins of the two systems and the hypothesized connections between them. GASS has been debated either to originate from a part (e.g., warp) of the Galactic disk or tidal debris of a disrupted Milky Way (MW) satellite galaxy. Our exploration shows that GASS is indeed made of stars from a dwarf spheroidal (dSph) galaxy, although we still can not rule out the possibility that GASS was dynamically created out of a previously formed outer MW disk. And whereas the TriAnd Star Cloud has been assumed to come from the tidal disruption of the same accreted MW satellite as the GASS/Monoceros Ring, our comparison of the abundance patterns in GASS and TriAnd M giants suggests that the TriAnd Star Cloud is likely an independent halo substructure unrelated to the GASS/Monoceros Ring. Furthermore, our findings also suggest that the MW may have accreted other satellites in addition to the on-going, well-known Sagittarius (Sgr) dwarf galaxy.
\end{abstract}

